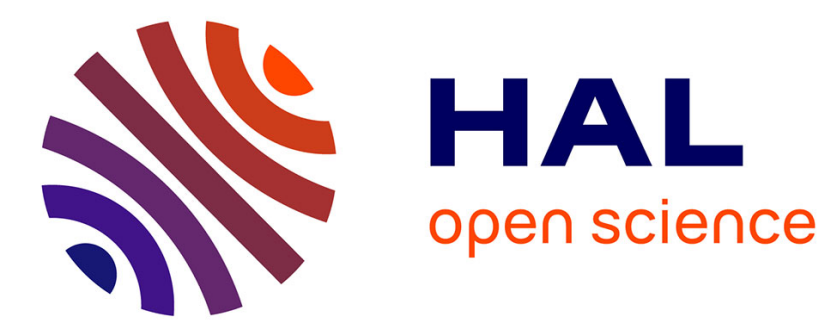

\title{
On the probabilities of hierarchical watersheds
}

Deise Santana Maia, Jean Cousty, Laurent Najman, Benjamin Perret

\section{To cite this version:}

Deise Santana Maia, Jean Cousty, Laurent Najman, Benjamin Perret. On the probabilities of hierarchical watersheds. International Symposium on Mathematical Morphology, Jul 2019, Saarbrücken, Germany. 10.1007/978-3-030-20867-7_11 . hal-02180484

\section{HAL Id: hal-02180484 https://hal.science/hal-02180484}

Submitted on 11 Jul 2019

HAL is a multi-disciplinary open access archive for the deposit and dissemination of scientific research documents, whether they are published or not. The documents may come from teaching and research institutions in France or abroad, or from public or private research centers.
L'archive ouverte pluridisciplinaire HAL, est destinée au dépôt et à la diffusion de documents scientifiques de niveau recherche, publiés ou non, émanant des établissements d'enseignement et de recherche français ou étrangers, des laboratoires publics ou privés. 


\title{
On the probabilities of hierarchical watersheds
}

\author{
Deise S. Maia, Jean Cousty, Laurent Najman, and Benjamin Perret \\ Université Paris-Est, LIGM (UMR 8049), CNRS, ENPC, ESIEE Paris, UPEM, \\ F-93162, Noisy-le-Grand, France
}

\begin{abstract}
Hierarchical watersheds are obtained by iteratively merging the regions of a watershed segmentation. In the watershed segmentation of an image, each region contains exactly one (local) minimum of the original image. Therefore, the construction of a hierarchical watershed of any image $I$ can be guided by a total order $\prec$ on the set of minima of $I$. The regions that contain the least minima according to the order $\prec$ are the first regions to be merged in the hierarchy. In fact, given any image $I$, for any hierarchical watershed $\mathcal{H}$ of $I$, there exists more than one total order on the set of minima of $I$ which could be used to obtain $\mathcal{H}$. In this article, we define the probability of a hierarchical watershed $\mathcal{H}$ as the probability of $\mathcal{H}$ to be the hierarchical watershed of $I$ for an arbitrary total order on the set of minima of $I$. We introduce an efficient method to obtain the probability of hierarchical watersheds and we provide a characterization of the most probable hierarchical watersheds.
\end{abstract}

\section{Introduction}

Watershed $[3,4]$ is a well established segmentation technique in the field of mathematical morphology. The idea underlying this technique comes from the topographic definition of watersheds: dividing lines between neighboring catchment basins, i.e., regions whose collected water drains to a common point. We say that the point (or region) of lowest altitude of a catchment basin is a (local) minimum of a topographic surface. In the context of digital image processing, gray-level images can be treated as topographic surfaces whose altitudes are determined by the pixel gray-levels. The local minima of an image are the regions of uniform grey-level surrounded by pixels of strictly higher gray-levels. We show the representation of a gray-scale image with four local minima and a watershed segmentation in Figure 1(a) and (b), respectively.

Hierarchical watersheds are sequences of nested segmentations equivalent to filterings of an initial watershed segmentation. Let $I$ be an image. The construction of a hierarchical watershed of $I$ is often based on a criterion used to order the minima of $I$, as the area and the dynamics $[13,8]$. More specifically, given any total order $\prec$ on the set of minima of $I$, the hierarchical watershed of $I$ for $\prec$ is constructed by iteratively "flooding" the minima of $I$ according to $\prec$. For instance, let us consider the total order $\prec$ on the set of minima $\{A, B, C, D\}$

This research is partly funded by the Bézout Labex, funded by ANR, reference ANR-10-LABX-58 


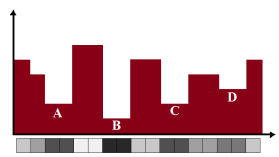

(a)

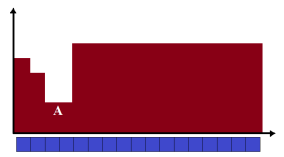

(e)

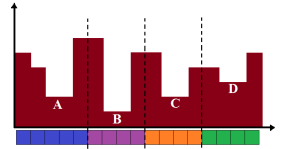

(b)

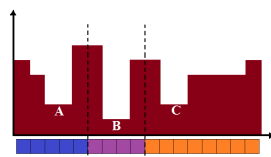

(f)

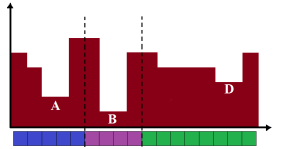

(c)

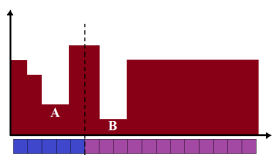

(g)

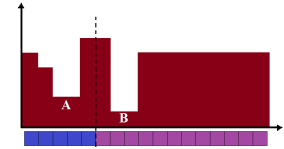

(d)

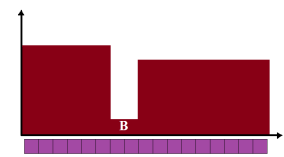

(h)

Fig. 1. (a): A gray-scale image $I$ with four minima. (b) A watershed segmentation of $I$ : the vertical dashed lines represent the watershed lines. (c), (d) and (e): The watershed segmentations resulting from iteratively flooding the minima $C, D$ and $B$, respectively. (f), (g) and (h): The watershed segmentations resulting from iteratively flooding the minima $D, C$ and $A$, respectively.

of the image $I$ of Figure 1(a) such that $C \prec D \prec B \prec A$. In Figure 1(b), (c), (d) and (e), we show the sequence of floodings of the minima of $I$ for $\prec$. The watershed segmentation of those floodings are the segmentations of the hierarchical watershed of $I$ for $\prec$.

In fact, we may obtain the same hierarchical watershed for several total orders on the set of minima of an image. For example, we show in Figure 1(b), (f), (g) and (h) the floodings of the minima of the image $I$ for another total order $\prec^{\prime}$ such that $D \prec^{\prime} C \prec^{\prime} A \prec^{\prime} B$. We can observe that the floodings for the total orders $\prec^{\prime}$ and $\prec$ induce the same sequence of watershed segmentations. Indeed, given any image $I$ and any hierarchical watershed $\mathcal{H}$ of $I$, there may exist several total orders on the set of minima of $I$ whose hierarchical watersheds correspond to $\mathcal{H}$. In other words, it is possible to order the minima of $I$ according to distinct criteria and still obtain the same hierarchical watershed.

In this article, (the gradients of) images are represented as weighted graphs. We define the probability of a hierarchical watershed $\mathcal{H}$ as the probability of $\mathcal{H}$ to be the hierarchical watershed of a given weighted graph $(G, w)$ for an arbitrary sequence of minima of $w$. Our contributions are twofold: (1) an efficient method to obtain the probability of hierarchical watersheds; and (2) a characterization of the most probable hierarchical watersheds of any weighted graph.

Other studies related to probability and (watershed) segmentations are found in $[1,2,12,9]$. In [1], a stochastic watershed segmentation based on random markers is introduced. In [2], the definitions of watersheds with multiple solutions for a single image are unified in the definition of tie-zone watersheds, which returns a unique solution. In [12], the authors propose a method to list the $k$-minimum spanning trees that induce distinct segmentations for a given set of markers. In [9], the authors estimate the probability that any two regions of a watershed segmentation have the same texture, which is further used to build hierarchies of segmentations. 
This paper is organized as follows. In section 2, we review graphs, hierarchies of partitions and saliency maps. In section 3, we propose an efficient method to compute the probability of hierarchical watersheds and we characterize the most probable hierarchical watersheds of any weighted graph.

\section{Background notions}

In this section, we first introduce hierarchies of partitions. Then, we review the definition of graphs, connected hierarchies and saliency maps. Subsequently, we define hierarchical watersheds.

\subsection{Hierarchies of partitions}

Let $V$ be a set. A partition of $V$ is a set $\mathbf{P}$ of non empty disjoint subsets of $V$ whose union is $V$. If $\mathbf{P}$ is a partition of $V$, any element of $\mathbf{P}$ is called a region of $\mathbf{P}$. Let $V$ be a set and let $\mathbf{P}_{1}$ and $\mathbf{P}_{2}$ be two partitions of $V$. We say that $\mathbf{P}_{1}$ is a refinement of $\mathbf{P}_{2}$ if every element of $\mathbf{P}_{1}$ is included in an element of $\mathbf{P}_{2}$. A hierarchy (of partitions on $V$ ) is a sequence $\mathcal{H}=\left(\mathbf{P}_{0}, \ldots, \mathbf{P}_{n}\right)$ of partitions of $V$ such that $\mathbf{P}_{i-1}$ is a refinement of $\mathbf{P}_{i}$, for any $i \in\{1, \ldots, n\}$ and such that $\mathbf{P}_{n}=\{V\}$. For any $i$ in $\{0, \ldots, n\}$, any region of the partition $\boldsymbol{P}_{i}$ is called a region of $\mathcal{H}$.

Hierarchies of partitions can be represented as trees whose nodes correspond to regions, as shown in Figure 2(a). Given a hierarchy $\mathcal{H}$ and two regions $X$ and $Y$ of $\mathcal{H}$, we say that $X$ is a parent of $Y$ (or that $Y$ is a child of $X$ ) if $Y \subset X$ and if $X$ is minimal for this property. In other words, if $X$ is a parent of $Y$ and if there is a region $Z$ such that $Y \subseteq Z \subset X$, then $Y=Z$. It can be seen that any region $X$ of $\mathcal{H}$ such that $X \neq V$ has exactly one parent. Thus, for any region $X$ such that $X \neq V$, we write $\operatorname{parent}(X)=Y$ where $Y$ is the unique parent of $X$. Given any region $R$ of $\mathcal{H}$, if $R$ is not the parent of any region of $\mathcal{H}$, we say that $R$ is a leaf region of $\mathcal{H}$. Otherwise, we say that $R$ is a non-leaf region of $\mathcal{H}$.

In Figure 2(a), the regions of a hierarchy $\mathcal{H}$ are linked to their parents and children by straight lines. The partition $\mathbf{P}_{0}$ of $\mathcal{H}$ contains all the leaf regions of $\mathcal{H}$.

\subsection{Graphs, connected hierarchies and saliency maps}

A graph is a pair $G=(V, E)$, where $V$ is a finite set and $E$ is a set of pairs of distinct elements of $V$, i.e., $E \subseteq\{\{x, y\} \subseteq V \mid x \neq y\}$. Each element of $V$ is called a vertex (of $G$ ), and each element of $E$ is called an edge (of $G$ ). To simplify the notations, the set of vertices and edges of a graph $G$ will be also denoted by $V(G)$ and $E(G)$, respectively.

Let $G=(V, E)$ be a graph and let $X$ be a subset of $V$. A sequence $\pi=$ $\left(x_{0}, \ldots, x_{n}\right)$ of elements of $X$ is a path (in $\left.X\right)$ from $x_{0}$ to $x_{n}$ if $\left\{x_{i-1}, x_{i}\right\}$ is an edge of $G$ for any $i$ in $\{1, \ldots, n\}$. The subset $X$ of $V$ is said to be connected for $G$ if, for any $x$ and $y$ in $X$, there exists a path from $x$ to $y$. The subset $X$ of $V$ is a connected component of $G$ if $X$ is connected and if, for any connected subset $Y$ 


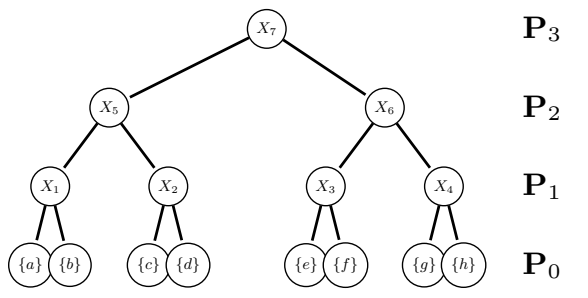

(a)

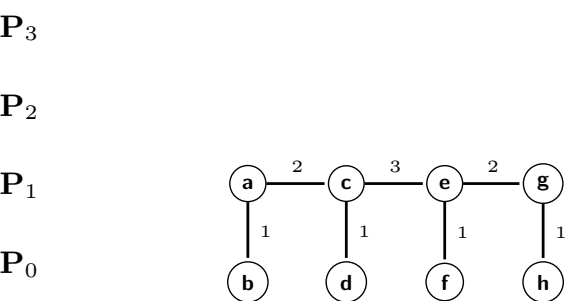

(b)

Fig. 2. (a): A representation of a hierarchy of partitions $\mathcal{H}=\left(\mathbf{P}_{0}, \mathbf{P}_{1}, \mathbf{P}_{2}, \mathbf{P}_{3}\right)$ on the set $\{a, b, c, d, e, f, g, h\}$. (b): A weighted graph $(G, w)$.

of $V$, if $X \subseteq Y$, then we have $X=Y$. In the following, we denote by $C C(G)$ the set of all connected components of $G$. It is well known that this set $C C(G)$ of all connected components of $G$ is a partition of the set $V$.

Let $G=(V, E)$ be a graph. A partition of $V$ is connected for $G$ if each of its regions is connected for $G$ and a hierarchy on $V$ is connected (for $G$ ) if any of its partitions is connected. For example, the hierarchy of Figure 2(a) is connected for the graph of Figure 2(b).

Let $G$ be a graph. If $w$ is a map from the edge set of $G$ to the set $\mathbb{R}^{+}$of positive real numbers, then the pair $(G, w)$ is called an (edge) weighted graph. If $(G, w)$ is a weighted graph, for any edge $u$ of $G$, the value $w(u)$ is called the weight of $u$ (for $w$ ).

As established in [6], a connected hierarchy can be equivalently defined with a weighted graph through the notion of a saliency map. Given a weighted graph $(G, w)$ and a hierarchy $\mathcal{H}=\left(\mathbf{P}_{0}, \ldots, \mathbf{P}_{n}\right)$ connected for $G$, the saliency map of $\mathcal{H}$ is the map from $E(G)$ to $\{0, \ldots, n\}$, denoted by $\Phi(\mathcal{H})$, such that, for any edge $u=\{x, y\}$ in $E(G)$, the value $\Phi(\mathcal{H})(u)$ is the smallest value $i$ in $\{0, \ldots, n\}$ such that $x$ and $y$ belong to a same region of $\mathbf{P}_{i}$. Therefore, the weight $\Phi(\mathcal{H})(u)$ of any edge $u=\{x, y\}$ is the ultrametric distance between $x$ and $y$ on the hierarchy $\mathcal{H}$. It follows that any connected hierarchy has a unique saliency map. Moreover, we can recover any hierarchy $\mathcal{H}$ connected for $G$ from the saliency map $\Phi(\mathcal{H})$ of $\mathcal{H}$. For instance, the weight map depicted in Figure $2(\mathrm{~b})$ is the saliency map of the hierarchy of Figure $2(\mathrm{a})$.

\subsection{Hierarchies of minimum spanning forests and watersheds}

In [4], the authors formalize watersheds in the framework of weighted graphs and show the optimality of watersheds in the sense of minimum spanning forests. In this section, we present hierarchical watersheds following the definition of hierarchies of minimum spanning forests presented in [7].

Let $G$ be a graph. We say that $G$ is a forest if, for any edge $u$ in $E(G)$, the number of connected components of the graph $(V(G), E(G) \backslash\{u\})$ is greater than the number of connected components of $G$. Given another graph $G^{\prime}$, we say 
that $G^{\prime}$ is a subgraph of $G$, denoted by $G^{\prime} \sqsubseteq G$, if $V\left(G^{\prime}\right) \subseteq V(G)$ and $E\left(G^{\prime}\right) \subseteq$ $E(G)$. Let $(G, w)$ be a weighted graph and let $G^{\prime}$ be a subgraph of $G$. A graph $G^{\prime \prime}$ is a Minimum Spanning Forest (MSF) of $G$ rooted in $G^{\prime}$ if:

1. $G^{\prime}$ is a subgraph of $G^{\prime \prime}$; and

2. the graphs $G$ and $G^{\prime \prime}$ have the same set of vertices, i.e., $V\left(G^{\prime \prime}\right)=V(G)$; and

3 . each connected component of $G^{\prime \prime}$ includes exactly one connected component of $G^{\prime}$; and

4. the sum of the weight of the edges of $G^{\prime \prime}$ is minimal among all graphs for which the above conditions 1,2 and 3 hold true.

Important notations and notions: in the sequel of this article, the symbol $G$ denotes a tree, i.e., a forest with a unique connected component. This implies that any map from $E(G)$ into $\mathbb{Z}^{+}$is the saliency map of a hierarchy which is connected for $G$. To shorten the notation, the vertex set of $G$ is denoted by $V$ and its edge set is denoted by $E$. The symbol $w$ denotes a map from $E$ into $\mathbb{R}^{+}$ such that, for any pair of distinct edges $u$ and $v$ in $E$, we have $w(u) \neq w(v)$. Thus, the pair $(G, w)$ is a weighted graph. Every hierarchy considered in this article is connected for $G$ and therefore, for the sake of simplicity, we use the term hierarchy instead of hierarchy which is connected for $G$.

Intuitively, a drop of water on a topographic surface drains in the direction of a local minimum and there is a correspondence between the catchment basins of a surface and its local minima. As established in [4], in the context of cuts, a notion of watershed in the framework of edge-weighted graphs is characterized as a (graph) cut induced by a minimum spanning forest rooted in the minima of this graph. Let $k$ be a value in $\mathbb{R}^{+}$. A connected subgraph $G^{\prime}$ of $G$ is a (regional) minimum (for $w$ ) at level $k$ if:

1. $G^{\prime}$ has at least one edge: $E\left(G^{\prime}\right) \neq \emptyset$; and

2. for any edge $u$ in $E\left(G^{\prime}\right)$, the weight of $u$ is equal to $k$; and

3. for any edge $\{x, y\}$ in $E(G) \backslash E\left(G^{\prime}\right)$ such that $\left|\{x, y\} \cap V\left(G^{\prime}\right)\right| \geq 1,\{x, y\}$ has a weight greater than $k$.

One can note that, since the weights of the edges of $G$ are pairwise distinct, it follows that any minimum of $G$ is a graph with a single edge.

Then, we follow the definition of hierarchical watersheds proposed in [5] which are optimal in the sense of minimum spanning forests. The sequence $\left(M_{1}, \ldots, M_{n}\right)$ of pairwise distinct subgraphs of $G$ is a sequence of minima of $w$ if $M_{i}$ is a minimum of $w$ for any $i \in\{1, \ldots, n\}$ and if $n$ is equal to the number of minima of $w$. In other words, a sequence of minima of $w$ is equivalent to a total order on the set of minima of $w$. We denote by $\mathcal{M}_{w}$ the set of all sequences of minima of $w$.

Definition 1 (hierarchical watershed) Let $\mathcal{S}=\left(M_{1}, \ldots, M_{n}\right)$ be a sequence of minima of $w$. Let $\left(G_{0}, \ldots, G_{n-1}\right)$ be a sequence of subgraphs of $G$ such that:

1. for any $i \in\{0, \ldots, n-1\}$, the graph $G_{i}$ is a MSF of $G$ rooted in $\left(\cup\left\{V\left(M_{j}\right) \mid\right.\right.$ $\left.j \in\{i+1, \ldots, n\}\}, \cup\left\{E\left(M_{j}\right) \mid j \in\{i+1, \ldots, n\}\right\}\right) ;$ and

2. for any $i \in\{1, \ldots, n-1\}, G_{i-1} \sqsubseteq G_{i}$. 


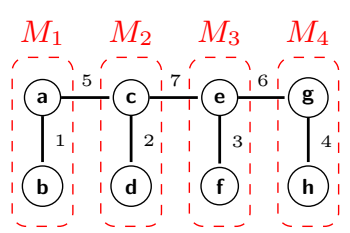

(a)

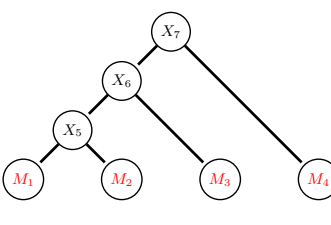

(b)

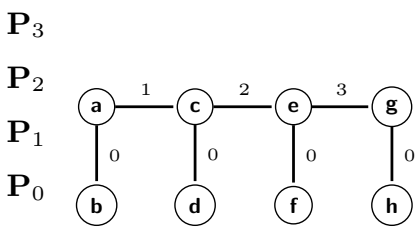

(c)

Fig. 3. (a): A weighted graph $(G, w)$ with four minima delimited by the dashed rectangles. (b): A hierarchical watershed $\mathcal{H}=\left(\mathbf{P}_{0}, \mathbf{P}_{1}, \mathbf{P}_{2}, \mathbf{P}_{3}\right)$ of $(G, w)$. (c): The saliency $\operatorname{map} \Phi(\mathcal{H})$ of $\mathcal{H}$.

The sequence $\mathcal{T}=\left(C C\left(G_{0}\right), \ldots, C C\left(G_{n-1}\right)\right)$ is called a hierarchical watershed of $(G, w)$ for $\mathcal{S}$. Given a hierarchy $\mathcal{H}$, we say that $\mathcal{H}$ is a hierarchical watershed of $(G, w)$ if there exists a sequence $\mathcal{S}$ of minima of $w$ such that $\mathcal{H}$ is a hierarchical watershed for $\mathcal{S}$.

A weighted graph $(G, w)$ and a hierarchical watershed $\mathcal{H}$ of $(G, w)$ are illustrated in Figure 3(a) and (b), respectively. We can see that $\mathcal{H}$ is the hierarchical watershed of $(G, w)$ for the sequence $\mathcal{S}=\left(M_{1}, M_{2}, M_{3}, M_{4}\right)$ of minima of $w$.

Since we assumed that the edge weights for $w$ are pairwise distinct, for any sequence $\mathcal{S}$ of minima of $w$, the hierarchical watershed of $(G, w)$ for $\mathcal{S}$ is unique.

\section{Studying probabilities of hierarchical watersheds}

Let $\mathcal{H}$ be a hierarchical watershed of $(G, w)$. In this study, we tackle the following problems: $\left(P_{1}\right)$ Find the probability of $\mathcal{H}$ to be the hierarchical watershed of $(G, w)$ for an arbitrary sequence of minima of $w$; and $\left(P_{2}\right)$ Characterize the most probable hierarchical watersheds of $(G, w)$.

In this section, we first introduce binary partition hierarchies by altitude ordering, which are closely linked to hierarchical watersheds, as established in [7]. Then, we present our solution to Problem $\left(P_{1}\right)$ and a quasi-linear time algorithm to compute probabilities of hierarchical watersheds. Finally, we propose a solution to Problem $\left(P_{2}\right)$.

\subsection{Binary partition hierarchies (by altitude ordering)}

Binary partition trees are widely used for hierarchical image representation [11]. In this section, we describe the particular case where the merging process is guided by the edge weights [7].

Given any set $X$, we denote by $|X|$ the number of elements of $X$. Let $k$ be any element in $\{1, \ldots,|E|\}$. We denote by $u_{k}$ the edge in $E$ such that there are $k-1$ edges in $E$ of weight strictly smaller than $w\left(u_{k}\right)$. We set $\mathbf{B}_{0}=\{\{x\} \mid x \in V\}$. The $k$-partition of $V$ is defined by $\mathbf{B}_{k}=\left(\mathbf{B}_{k-1} \backslash\left\{\mathbf{B}_{k-1}^{x}, \mathbf{B}_{k-1}^{y}\right\}\right) \cup\left\{\mathbf{B}_{k-1}^{y} \cup \mathbf{B}_{k-1}^{x}\right\}$, 


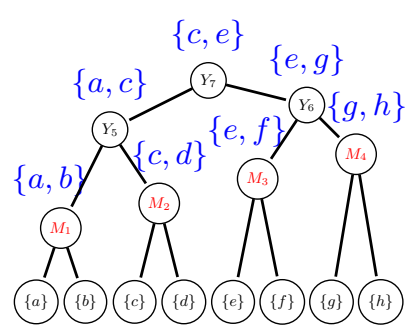

(a)

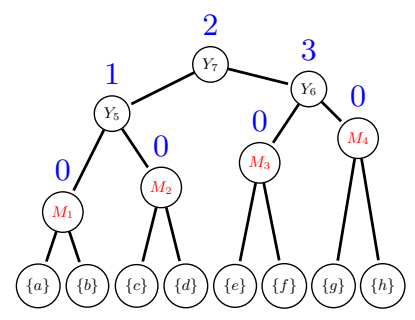

(b)

Fig. 4. (a) The binary partition hierarchy $\mathcal{B}$ of $(G, w)$ of Figure 3(a). (b) A representation of the saliency map $\Phi(\mathcal{H})$ of Figure $3(\mathrm{c})$.

where $u_{k}=\{x, y\}$ and $\mathbf{B}_{k-1}^{x}$ and $\mathbf{B}_{k-1}^{y}$ are the regions of $\mathbf{B}_{k-1}$ that contain $x$ and $y$, respectively. The binary partition hierarchy (by altitude ordering) of $(G, w)$, denoted by $\mathcal{B}$, is the hierarchy $\left(\mathbf{B}_{0}, \ldots, \mathbf{B}_{|E|}\right)$.

Let $\mathcal{B}=\left(\mathbf{B}_{0}, \ldots, \mathbf{B}_{|E|}\right)$ be the binary partition hierarchy of $(G, w)$ and let $k$ be a value in $\{1, \ldots,|E|\}$. Since $G$ is a tree, given $u_{k}=\{x, y\}$, it can be seen that $\mathbf{B}_{k-1}^{x}$ and $\mathbf{B}_{k-1}^{y}$ are disjoint. Thus $\mathbf{B}_{k}$ is different from $\mathbf{B}_{k-1}$. We can affirm that $\mathbf{B}_{k-1}^{x} \cup \mathbf{B}_{k-1}^{y}$ is not a region of $\mathbf{B}_{k^{\prime}}$ for any $k^{\prime}<k$. Since $\mathcal{B}$ is a hierarchy, we say that $u_{k}$ is the building edge of the region $\left\{\mathbf{B}_{k-1}^{y} \cup \mathbf{B}_{k-1}^{x}\right\}$ of $\mathcal{B}$. Given any edge $u$ in $E$, we denote by $R_{u}$ the region of $\mathcal{B}$ whose building edge is $u$.

In Figure 4(a), we present the binary partition hierarchy $\mathcal{B}$ of the graph $(G, w)$ of Figure 3(a). The building edge of each non-leaf region $R$ of $\mathcal{B}$ is shown above the node that represents $R$.

\subsection{Finding probabilities of hierarchical watersheds}

Let $\mathcal{H}$ be a hierarchical watershed of $(G, w)$. As defined previously, there is a sequence $\mathcal{S}$ of minima of $w$ such that $\mathcal{H}$ is the hierarchical watershed of $(G, w)$ for $\mathcal{S}$. Indeed, as illustrated in Figure 1, the hierarchy $\mathcal{H}$ may be the hierarchical watershed of $(G, w)$ for several sequences of minima of $w$. Thus, for any hierarchical watershed $\mathcal{H}$ of $(G, w)$, we denote by $S_{w}(\mathcal{H})$ the set which contains every sequence $S$ of minima such that the hierarchical watershed of $(G, w)$ for $\mathcal{S}$ is $\mathcal{H}$.

Definition 2 (probability of a hierarchical watershed) Let $\mathcal{H}$ be a hierarchical watershed of $(G, w)$. Let $\mathcal{S}$ be uniformely distributed on the set $\mathcal{M}_{w}$ of all sequences of minima of $w$. We define the probability of $\mathcal{H}$ knowing $w$, denoted by $p(\mathcal{H} \mid w)$, as the probability that the hierarchical watershed of $(G, w)$ for $\mathcal{S}$ is equal to $\mathcal{H}$.

Property 3 Let $\mathcal{H}$ be a hierarchical watershed of $(G, w)$. The probability $p(\mathcal{H} \mid w)$ of $\mathcal{H}$ knowing $w$ is the ratio $k / n$ where $k$ and $n$ are the numbers of elements of $S_{w}(\mathcal{H})$ and of $\mathcal{M}_{w}$, respectively.

In order to solve the problem of finding the probability of hierarchical watersheds, we first introduce watershed-cut edges and maximal regions. 
Definition 4 (watershed-cut edge) Let $\mathcal{H}$ be a hierarchical watershed of $(G, w)$ and let $\mathbf{P}$ be the set of leaf regions of $\mathcal{H}$. Let $u=\{x, y\}$ be an edge of $G$. If $x$ and $y$ belong to distinct regions of $\mathbf{P}$, we say that $u$ is a watershed-cut edge of $w$.

We can observe that any hierarchical watershed of $(G, w)$ has the same set of leaf regions. For example, the set of watershed-cut edges of the graph $(G, w)$ of Figure 3(a) is $\{\{a, c\},\{c, e\},\{e, g\}\}$, which is the set of edges whose vertices are in distinct leaf regions of the hierarchical watershed $\mathcal{H}$ of Figure 3(b).

Definition 5 (maximal region) Let $u$ be a watershed-cut edge of $w$ and let $f$ be a map from $E$ into $\mathbb{R}^{+}$. Let $R_{u}$ be the region of $\mathcal{B}$ whose building edge is $u$. We say that the region $R_{u}$ is a maximal region of $\mathcal{B}$ for $f$ if the value of $f$ on the building edge $u$ of $R_{u}$ is greater than the value of $f$ on the building edge of any region of $\mathcal{B}$ included in $R_{u}$ : i.e., if $f(u)>\max \left\{f(v), v \in E \mid R_{v} \subset R_{u}\right\}$.

In Figure 4(b), we represent the saliency map $\Phi(\mathcal{H})$ of Figure 3(c) on the binary partition hierarchy by altitude ordering $\mathcal{B}$ of Figure $4(\mathrm{a})$. The weight above each region $R$ of $\mathcal{B}$ is the weight of the building edge of $R$ for $\Phi(\mathcal{H})$. We can see that the only maximal regions of $\mathcal{B}$ for $\Phi(\mathcal{H})$ are the regions $Y_{5}$ and $Y_{6}$.

The following property establishes that, given any hierarchical watershed $\mathcal{H}$ of $(G, w)$, the probability of $\mathcal{H}$ knowing $w$ can be defined through the number of maximal regions of $\mathcal{B}$ for the saliency map $\Phi(\mathcal{H})$ of $\mathcal{H}$.

Property 6 Let $\mathcal{H}$ be a hierarchical watershed of $(G, w)$ and let $m$ be the number of maximal regions of $\mathcal{B}$ for the saliency map $\Phi(\mathcal{H})$ of $\mathcal{H}$. The probability of $\mathcal{H}$ knowing $w$ is

$$
p(\mathcal{H} \mid w)=\frac{2^{m}}{\left|\mathcal{M}_{w}\right|} .
$$

For instance, let us consider the hierarchical watershed $\mathcal{H}$ of Figure 3(b). As stated previously, the hierarchy $\mathcal{B}$ has two maximal regions $\left(Y_{5}\right.$ and $\left.Y_{6}\right)$ for $\Phi(\mathcal{H})$. Since the graph $(G, w)$ of Figure 3 has four minima, there are 4 ! sequences of minima of $w$. By property 6 , we may conclude that the probability of $\mathcal{H}$ knowing $w$ is $\frac{2^{2}}{4 !}$. Indeed, $\mathcal{H}$ is the hierarchical watershed of $(G, w)$ for four sequences of minima: $\left(M_{1}, M_{2}, M_{3}, M_{4}\right),\left(M_{1}, M_{2}, M_{4}, M_{3}\right),\left(M_{2}, M_{1}, M_{3}, M_{4}\right)$ and $\left(M_{2}, M_{1}, M_{4}, M_{3}\right)$.

From Property 6, we derive a quasi-linear time algorithm (Algorithm 1) to compute the probability of hierarchical watersheds. The inputs are a weighted graph $((V, E), w)$ and the saliency map $f$ of a hierarchical watershed of $((V, E), w)$. First, the binary partition hierarchy $\mathcal{B}$ of $((V, E), w)$ is computed in quasi-linear time with respect to $|E|[10]$. At lines $2-7$, we compute the number of minima of $w$ and the set of watershed-cut edges of $w$ in linear time with respect to $|E|$ using the method proposed in [10]. At lines $8-20$, we find the number $m$ of maximal regions of $\mathcal{B}$ for $f$. Both for loops at lines 8 and 15 can be computed in linear time with respect to $|E|$. Therefore, the overall time complexity of Algorithm 1 is quasi-linear with respect to $|E|$. The output of Algorithm 1 is the probability of $\mathcal{H}$ knowing $w$, which is $\frac{2^{m}}{n u m M i n !}$ by Property 6 . 


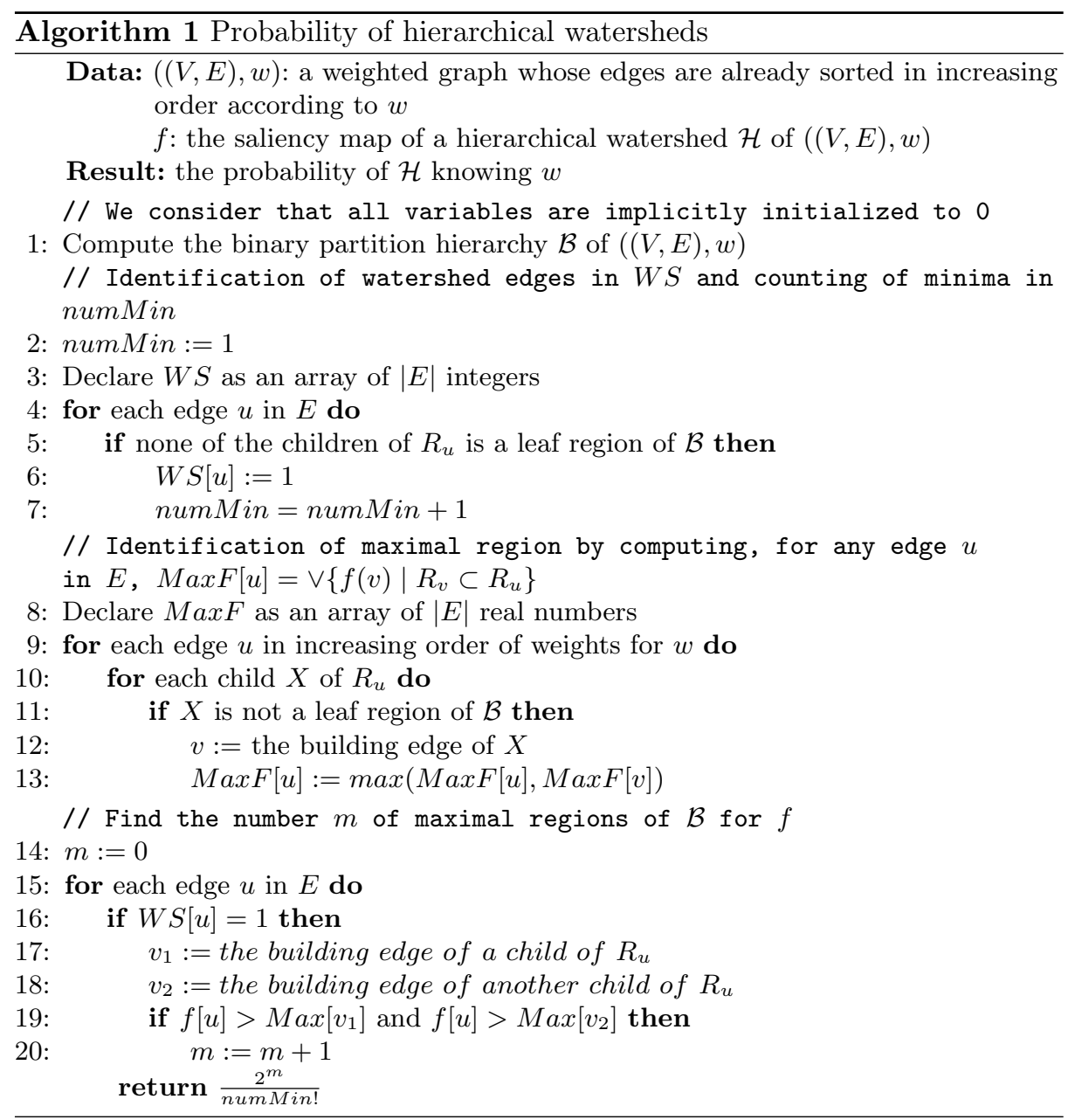

\subsection{Most probable hierarchical watersheds}

Let $\ell$ be the number of watershed-cut edges of $w$. We can affirm that there are at most $\ell$ maximal regions of $\mathcal{B}$ for the saliency map of any hierarchical watershed of $(G, w)$. Thus, we can derive the following Corollary 7 , which establishes the tight upper bound on the probability of any hierarchical watershed of $(G, w)$.

Corollary 7 Let $\ell$ be the number of watershed-cut edges of $w$ and let $\mathcal{H}$ be a hierarchical watershed of $(G, w)$. The tight upper bound on the probability of $\mathcal{H}$ knowing $w$ is $\frac{2^{\ell}}{\left|\mathcal{M}_{w}\right|}$.

Let $u$ be a watershed-cut edge of $w$. We say that $R_{u}$ is a primary region of $\mathcal{B}$ if there is no watershed-cut edge $v$ of $w$ such that $R_{v} \subset R_{u}$. Let $f$ be the saliency map of a hierarchical watershed of $(G, w)$. One can note that the value of $f$ is 
null on non watershed-cut edges of $w$ and non null on the watershed-cut edges of $w$. Therefore, given a watershed-cut edge $u$ of $w$, if $R_{u}$ is a primary region of $\mathcal{B}$, then $f(u)>\max \left\{f(v), v \in E \mid R_{v} \subset R_{u}\right\}=0$ and, consequently, $R_{u}$ is a maximal region of $\mathcal{B}$ for $f$. We conclude that each primary region of $\mathcal{B}$ is a maximal region of $\mathcal{B}$ for every saliency map of a hierarchical watershed of $(G, w)$. We can now define the tight lower bound on the probability of hierarchical watersheds.

Corollary 8 Let $k$ be the number of primary regions of $\mathcal{B}$ and let $\mathcal{H}$ be a hierarchical watershed of $(G, w)$. The tight lower bound on the probability of $\mathcal{H}$ knowing $w$ is $\frac{2^{k}}{\left|\mathcal{M}_{w}\right|}$.

If the map $w$ has more than two minima, then there is at least one watershedcut edge $u$ of $w$ such that $R_{u}$ is not a primary region of $\mathcal{B}$. Therefore, the tight lower bound and the tight upper bound on the probabilities of hierarchical watersheds of $(G, w)$ are not equal. This justifies the following definition of most probable hierarchical watersheds.

Definition 9 (most probable hierarchical watersheds) Let $\mathcal{H}$ be a hierarchical watershed of $(G, w)$. We say that $\mathcal{H}$ is a most probable hierarchical watershed of $(G, w)$, if, for any hierarchical waterhsed $\mathcal{H}^{\prime}$ for $(G, w)$, we have $p(\mathcal{H} \mid$ $w) \geq p\left(\mathcal{H}^{\prime} \mid w\right)$.

Let $f$ be the saliency map of a hierarchical watershed of $(G, w)$. Let $R$ be a non-leaf region of $\mathcal{B}$ and let $u$ be the building edge of $R$. We define the persistence value of $R$ for $f$ as the weight $f(u)$ of $u$. Let $\mathcal{H}$ be a hierarchical watershed of $(G, w)$. By Corollary 7 , the probability of $\mathcal{H}$ knowing $w$ is maximal when, for every watershed-cut edge $u$ of $w, R_{u}$ is a maximal region of $\mathcal{B}$ for $\Phi(\mathcal{H})$. By the definition of maximal regions, we can establish the following characterization of the most probable hierarchical watersheds of $(G, w)$.

Property 10 Let $\mathcal{H}$ be a hierarchical watershed of $(G, w) . \mathcal{H}$ is a most probable hierarchical watershed of $(G, w)$ if and only if the persistence values for $\Phi(\mathcal{H})$ are increasing on the hierarchy $\mathcal{B}$.

Let $\mathcal{H}$ be a most probable hierarchical watershed of $(G, w)$. By Property 10 , we may conclude that the order in which the regions of $\mathcal{H}$ are merged along the partitions of $\mathcal{H}$ are constrained by the hierarchy $\mathcal{B}$. Thus, we can deduce the following corollary from Property 10.

Corollary 11 Let $\mathcal{H}$ be a hierarchical watershed of $(G, w) . \mathcal{H}$ is a most probable hierarchical watershed of $(G, w)$ if and only if each non-leaf region of $\mathcal{H}$ is a region of $\mathcal{B}$.

In Figure 5, we present four hierarchical watersheds for the weighted graph of Figure 3(a). Indeed, those are the only hierarchical watersheds for $(G, w)$. For each hierarchy, we present its saliency map represented on $G$ and on the binary 

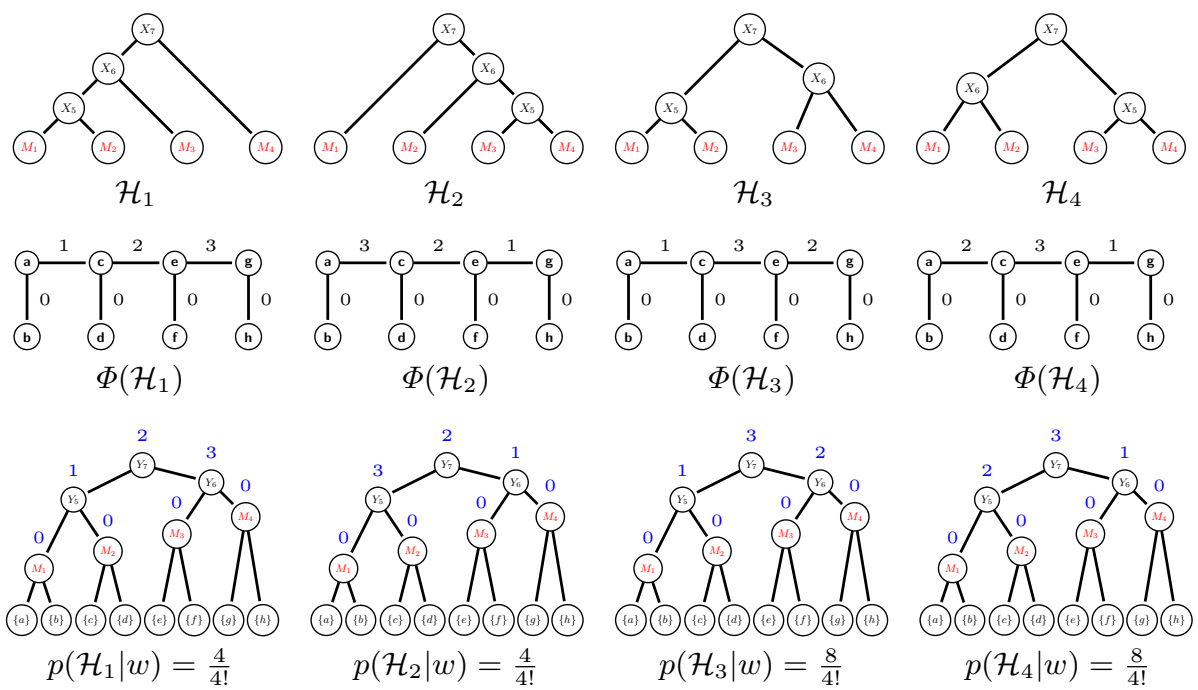

Fig. 5. The hierarchical watersheds for the weighted graph $(G, w)$ of Figure 3(a), their saliency maps represented on the graph $(G, w)$ and on the binary partition hierarchy $\mathcal{B}$. The probability of each hierarchical watershed knowing $w$ is presented under $\mathcal{B}$.

partition hierarchy by altitude ordering $\mathcal{B}$. Since $w$ has four minima, the number of sequences of minima of $w$ is 4 !. The probability of the hierarchies $\mathcal{H}_{1}, \mathcal{H}_{2}, \mathcal{H}_{3}$ and $\mathcal{H}_{4}$ knowing $w$ are $\frac{4}{4 !}, \frac{4}{4 !}, \frac{8}{4 !}$ and $\frac{8}{4 !}$, respectively. Therefore, the set of most probable hierarchical watersheds of $(G, w)$ is $\left\{\mathcal{H}_{3}, \mathcal{H}_{4}\right\}$. We can verify that each non-leaf region of $\mathcal{H}_{3}$ and $\mathcal{H}_{4}$ is a region of $\mathcal{B}$, as established by Corollary 11, which is not the case for $\mathcal{H}_{1}$ and $\mathcal{H}_{2}$.

From Property 10, we can deduce a recursive method to find the saliency map $f$ of a most probable hierarchical watershed of $(G, w)$. Let $\ell$ be the number of watershed-cut edges of $w$ and let $L$ be the set $\{1, \ldots, \ell\}$. Let $R$ be the root of the tree representing $\mathcal{B}$ and let $u$ be the building edge of $R$. First, we assign $f(u)$ to $\max \{1, \ldots, \ell\}$. Then, we divide the set $\{1, \ldots, \ell-1\}$ into two subsets $L^{\prime}$ and $L^{\prime \prime}$ with $n^{\prime}-1$ and $n^{\prime \prime}-1$ elements, respectively, where $n^{\prime}$ and $n^{\prime \prime}$ are the number of minima included in the children $R^{\prime}$ and $R^{\prime \prime}$ of $R$. Subsequently, the sets $L^{\prime}$ and $L^{\prime \prime}$ are propagated to $R^{\prime}$ and $R^{\prime \prime}$, respectively. The subtrees rooted in $R^{\prime}$ and $R^{\prime \prime}$ are treated separately. This process is performed until the weight of all watershed-cut edges of $w$ have been assigned. An illustration of this method is presented in Figure 6, where we show two of the most probable hierarchical watersheds of an image.

\section{Conclusion}

We proposed an efficient method to obtain the probability of hierarchical watersheds in the framework of weighted graphs. We also provided a characterization 


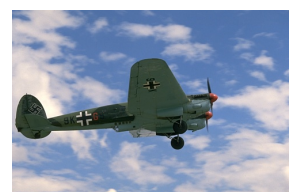

$I$

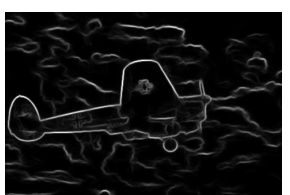

Grad

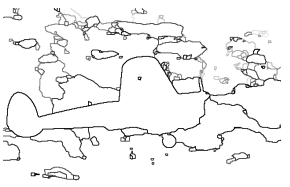

$f_{1}$

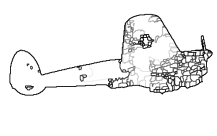

$f_{2}$

Fig. 6. An image $I$, a gradient $G r a d$ of $I$ and the saliency maps $f_{1}$ and $f_{2}$ of two of the most probable hierarchical watersheds of Grad.

of the most probable hierarchical watersheds of any weighted graph. In future work, we will extend the notions presented here to arbitrary graphs, i.e. graphs that are not trees and graphs whose edge weights are not pairwise distinct.

\section{References}

1. J. Angulo and D. Jeulin. Stochastic watershed segmentation. In ISMM. Springer, 2007.

2. R. Audigier and R. Lotufo. Uniquely-determined thinning of the tie-zone watershed based on label frequency. JMIV, 27(2):157-173, 2007.

3. S. Beucher and F. Meyer. The morphological approach to segmentation: the watershed transformation. Optical Engineering-New York-Marcel Dekker Incorporated-, 34:433-433, 1992.

4. J. Cousty, G. Bertrand, L. Najman, and M. Couprie. Watershed cuts: Minimum spanning forests and the drop of water principle. IEEE PAMI, 31(8):1362-1374, 2009.

5. J. Cousty and L. Najman. Incremental algorithm for hierarchical minimum spanning forests and saliency of watershed cuts. In ISMM, pages 272-283. Springer, 2011.

6. J. Cousty, L. Najman, Y. Kenmochi, and S. Guimarães. Hierarchical segmentations with graphs: quasi-flat zones, minimum spanning trees, and saliency maps. JMIV, 60(4):479-502, 2018.

7. J. Cousty, L. Najman, and B. Perret. Constructive links between some morphological hierarchies on edge-weighted graphs. In ISMM, pages 86-97. Springer, 2013.

8. M. Grimaud. New measure of contrast: the dynamics. In Image Algebra and Morphological Image Processing III, volume 1769, pages 292-306. International Society for Optics and Photonics, 1992.

9. D. Jeulin. Morphological probabilistic hierarchies for texture segmentation. Mathematical Morphology-Theory and Applications, 1(1), 2016.

10. L. Najman, J. Cousty, and B. Perret. Playing with kruskal: algorithms for morphological trees in edge-weighted graphs. In ISMM, pages 135-146. Springer, 2013.

11. P. Salembier and L. Garrido. Binary partition tree as an efficient representation for image processing, segmentation, and information retrieval. IEEE transactions on Image Processing, 9(4):561-576, 2000.

12. C. Straehle, S. Peter, U. Köthe, and F. A. Hamprecht. K-smallest spanning tree segmentations. In German Conference on Pattern Recognition, pages 375-384. Springer, 2013.

13. C. Vachier and F. Meyer. Extinction value: a new measurement of persistence. In IEEE Workshop on nonlinear signal and image processing, pages 254-257, 1995. 